\title{
Guest Editorial: new basic and clinical aspects of graft-versus-host disease: what can we do without "Doc" Brown's help?
}

\author{
Akiyoshi Takami
}

Received: 26 July 2013/Revised: 7 August 2013/Accepted: 7 August 2013/Published online: 20 August 2013

(c) The Japanese Society of Hematology 2013

When confronting a difficult case of graft-versus-host disease (GVHD), have you ever wished to be able to go back in time? I suppose that only those of you who already have a time machine, like the one operated by Emmett "Doc" Brown and his friend, Martin "Marty" McFly from the 1985 film Back to the Future can honestly say they have not.

Allogeneic hematopoietic stem cell transplantation (HSCT) offers a curative therapy for malignant and nonmalignant hematologic diseases and other disorders. However, GVHD remains a major and lethal complication that limits the wider application of allogeneic HSCT. GVHD develops in recipients of donor grafts that contain $\mathrm{T}$ cells responding to genetically distinct proteins on host cells. Human leukocyte antigen (HLA) genes, such as HLA-A, B, C, DRB1, DPB1, and DQB1, which have vast numbers of single nucleotide polymorphisms (SNPs), are the most important factor affecting the risk and severity of GVHD. Polymorphisms of non-HLA genes that mainly impact individual immune response to infections and inflammatory reactions are involved in GVHD as well, as evidenced by the fact that GVHD also sometimes occurs in 12/12 matched transplants.

The greatest concern in the treatment of GVHD is that steroids remain the first-line therapy for GVHD and no standard treatment strategy for patients refractory to steroid therapy exists. In other words, once GVHD has begun, it is already too late to effectively intervene. In Back to the Future, Doc Brown constructed a machine that enabled him to travel back in time to alter the course of future events. This allowed Marty McFly to eliminate the causes

\footnotetext{
A. Takami $(\bowtie)$

Department of Hematology and Oncology, Kanazawa University

Hospital, 13-1 Takaramachi, Kanazawa 920-8641, Japan

e-mail: takami@staff.kanazawa-u.ac.jp
}

of unfavorable events before they could take place. We are now faced with a similar situation in the management of GVHD. Without Doc Brown's help, what can be done? I hope this month's "Progress in Hematology" will provide readers with some fresh clues.

This issue includes three excellent review articles [1-3] in addition to my own [4], which focus on novel developments in the pathophysiology of acute GVHD, and on the prediction of GVHD using HLA and non-HLA polymorphisms and GVHD biomarkers. Dr. Junya Kanda summarizes findings from large cohort studies, including those he directed, to investigate the effect of HLA mismatch in each allele and antigen on the risk and severity of acute GVHD, and reports that the effect of HLA matching should be evaluated cautiously with respect to stem cell source, GVHD prophylaxis, the year of transplant, recipient age, ethnicity, and HLA distribution. This information should be useful in selecting an optimal donor in light of current practice.

In my review, I detail non-HLA gene polymorphisms associated with GVHD and their clinical significance, which may be useful in further analyses, including functional investigations and genome-wide association studies. Prior to HSCT, it is important to choose an optimal donor with an eye to achieve a lower risk of GVHD; high-resolution HLA genotyping for recipients and donor candidates is effective, while non-HLA genotyping may also provide useful information.

Dr. Yoshinobu Maeda reviews the importance of innate immune response in GVHD. HSCT is associated with cellular and tissue damage due to the use of cytotoxic agents, total body irradiation, infections, and so on, which can result in the release of exogenous and endogenous "danger signals", activating the innate immune response, which then triggers or amplifies acute GVHD. This review 
helps us to understand the importance of danger signals and microangiopathies in the pathogenesis of acute GVHD, information that may help open avenues into new therapeutic approaches or patient management strategies.

Dr. Sophie Paczesny, a pioneer and a top physicianscientist in this field, provides vast amounts of information on GVHD biomarkers, as well as novel knowledge and challenges, which are critically important for developing means of diagnosing GVHD early, precisely, and predictably, enabling optimal approaches to the prevention and management of this intractable complication in a patientspecific manner. The biomarkers clinically available for both acute and chronic GVHD can lead to dramatic improvements in the HSCT outcomes. The identification of GVHD biomarkers to predict the development, response to treatment, and prognosis of GVHD may allow for preemptive treatment strategies, rather than less-effective prophylactic and disease-oriented strategies, in a manner analogous to the way cytomegalovirus reactivation after HSCT is managed at present. Moreover, biomarkers that correlate with subsequent response to GVHD treatment may be able to determine when and how GVHD treatment is withdrawn, or when alternative therapy should be initiated.

The ultimate goal of all of these efforts is the elimination of GVHD-related mortality and morbidity without compromising the anti-tumor and anti-microbial immunity, substantially improving the safety and efficacy of allogeneic HSCT. The "Progress in Hematology" articles in this issue were written from this point of view. With the progress made thus far and ongoing efforts, these reviews will hopefully contribute to improved management of allogeneic HSCT recipients and help us to achieve our goal of preventing GVHD-related mortality and morbidity.

Acknowledgments This study was supported by grants from the Ministry of Health, Labour and Welfare of Japan, and the Ministry of Education, Culture, Sports and Technology of Japan. This work was supported in part by a Research on Allergic Disease and Immunology (H23-010) in a Health and Labor Science Grant from the Ministry of Health, Labour and Welfare of Japan. The grant sources played no role in the study design, data collection and analysis, the decision to publish or the preparation of the manuscript.

Conflict of interest The authors declare no competing financial interests.

\section{References}

1. Paczesny S, Raiker N, Brooks S, Mumaw C. Graft-versus-host disease biomarkers: omics and personalized medicine. Int $\mathrm{J}$ Hematol. 2013.

2. Maeda Y. Pathogenesis of graft versus host disease: innate immunity amplifying acute alloimmune responses. Int J Hematol 2013.

3. Kanda J. The role of HLA mismatch in graft-versus-host disease. Int J Hematol. 2013.

4. Takami A. The role of non-HLA gene polymorphisms in graftversus-host disease. Int J Hematol. 2013. 